\title{
Meningomyelocele: Early Detection Using 3- Dimensional Ultrasound Imaging in the Family Medicine Center
}

\author{
Christopher P. Forest, MSHS, PA-C, Darenie Goodman, MD, and \\ Ricardo G. Habn, MD
}

A young primigravida presented to the family medicine clinic 7 weeks pregnant. Standard 2-dimensional ultrasound at 9 weeks revealed a grossly abnormal posterior brain and 2 adjacent sonolucent structures: 2 yolk sacs versus a yolk sac and cyst. Imaging by 3-dimensional ultrasound distinguished these structures, revealing a caudal cyst with continuity of fetal tissue consistent with a meningomyelocele. To date there is no documentation in the literature of a meningomyelocele diagnosed during the first trimester of pregnancy. Identification of neural tube defects early in pregnancy offers increased options to the mother and may impact long-term fetal prognosis. (J Am Board Fam Med 2010;23:270-272.)

Keywords: Meningomyelocele, 3-Dimensional Ultrasound, First Trimester, Neural Tube Defect, Ultrasound, Congenital Defects, Pregnancy/Prenatal Care

A 24-year-old, African-American female presented to the family medicine clinic (FMC) with abdominal discomfort, intermittent nausea, and amenorrhea. She had been taking quetiapine intermittently for bipolar depression. Her other medications included ondansetron for occasional nausea, diphenhydramine for allergic rhinitis, and atropine/hyoscyamine/phenobarbital/scopolamine tablets as needed for ileitis. Family history was positive for diabetes mellitus on her mother's side and negative for neural tube defects.

Physical examination was unremarkable; however, urine human chorionic gonatropin was positive. Because this primigravida was unsure of the date of her last menstrual period, a diagnostic ultrasound was performed in the FMC, which confirmed an intra-

This article was externally peer reviewed.

Submitted 25 September 2009; revised 13 October 2009; accepted 19 October 2009.

From the Department of Family Medicine, Keck School of Medicine of USC (CPF, DG, RGH), and the Alfred Mann Institute for Biomedical Engineering (RGH), University of Southern California, Los Angeles.

Funding: none.

Conflict of interest: none declared.

Corresponding author: Christopher P. Forest, MSHS, PA-C, Family Medicine, Keck School of Medicine of USC, 1000 South Fremont Avenue, Unit 7, Building A6, 4th Floor, Room 6432, Alhambra, CA 91803 (E-mail: cforest@usc.edu). uterine pregnancy at 7 weeks 2 days, fetal heart rate of 152 beats per minute, and normal adnexal structures. Two weeks later the patient presented with vaginal bleeding and a repeat ultrasound revealed a grossly abnormal posterior brain and a single fetus with 2 adjacent, round, sonolucent structures, which were 2 yolk sacs versus a yolk and cyst (Figure 1). The patient had not been taking prenatal vitamins with folic acid or other nutritional supplements.

Further investigation of these structures revealed a caudal cyst adjacent to the fetus (Figure 2). Standard 2-dimensional ultrasound techniques were unable to distinguish whether this cystic structure at the base of the spine was an extension of fetal tissue or a separate structure. To make this determination in the FMC, a 3-dimensional ultrasound (Voluson 730, GE, Fairfield, CT) was performed. The results showed continuity of fetal tissue consistent with a meningomyelocele (MMC) (Figure 3). After discussion with both the patient and her mother, the patient elected to terminate the pregnancy. Direct visualization of the defect was not possible because of the aspiration process of dilatation and curettage.

\section{Discussion}

Neural tube defects are the second most common congenital abnormalities, second only to cardiac 
Figure 1. Standard 2-dimensional ultrasound imaging reveals a single fetal pole with what appears to be a dual yolk sac. The absence of a second pole and the difference in brightness between the 2 structures suggests that one may be a cyst rather than a second yolk sac.

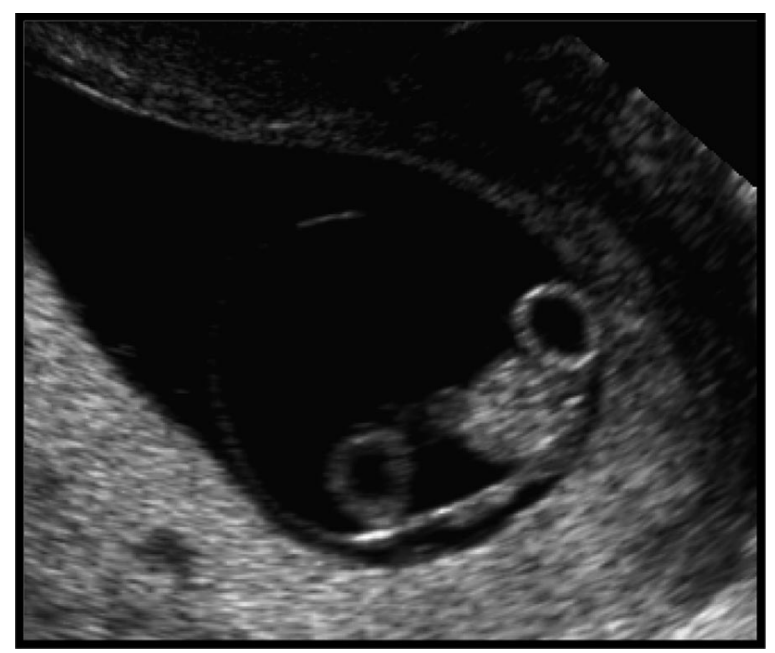

malformations. ${ }^{1}$ The most common nonlethal form of these anomalies ${ }^{2}$ is the MMC, a condition caused by failure of the neural tube to fuse completely by the end of the fourth week after conception, leaving meninges and neural tissue exposed through the vertebral arch and skin. This condition is estimated to occur in 1 of 2000 births in the

Figure 2. Standard 2-dimensional ultrasound imaging reveals an adjacent sonolucent cystic structure (arrow) at the base of the fetus. This technique cannot differentiate between a caudal cyst and a fetal abnormality.

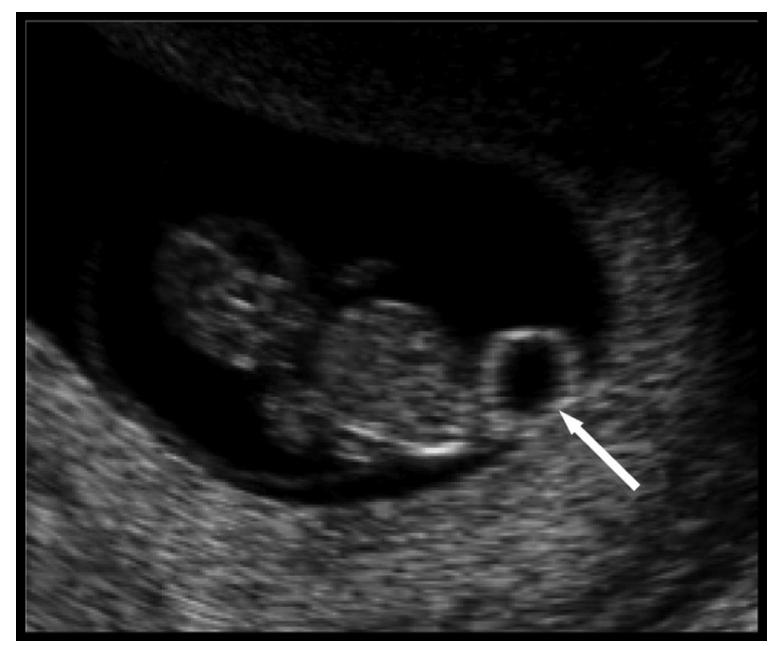

Figure 3. Advanced 3-dimensional ultrasound imaging demonstrates that this structure (arrow) is contiguous with the fetus and consistent with a meningomyelocele.

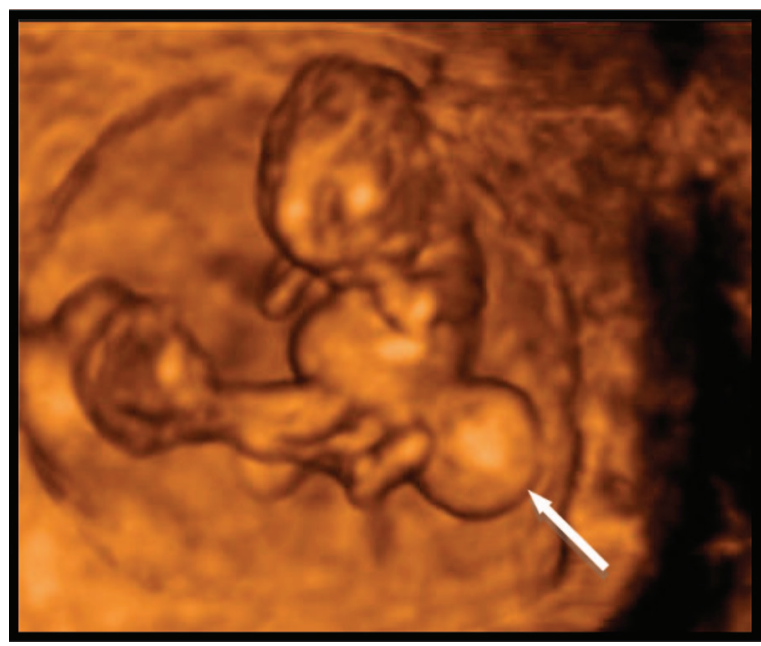

United States. ${ }^{2}$ Hydrocephalus, resulting from the Arnold Chiari II malformation, is a common prenatal and neonatal complication associated with MMC.

Seventy-five percent of the patients are estimated to reach adulthood with aggressive treatment. ${ }^{3}$ They suffer from various degrees of neurological deficits depending on the level of the lesion. Individuals with an L3 lesion or higher are not expected to walk. ${ }^{3}$ Urinary and fecal incontinence are common. An estimated $20 \%$ of these patients require special education classes and $30 \%$ have an intelligence quotient below 80 , secondary to hydrocephalus. ${ }^{3}$

The American College of Obstetricians and Gynecologists recommends maternal serum $\alpha$-fetoprotein evaluation at 15 to 20 weeks' gestation to screen for neural tube defects. ${ }^{4}$ This testing is limited by a high rate of false positives. A true positive results from a spinal fluid leak at the site the deformity. Amniocentesis can be performed to establish the diagnosis, a procedure that carries a 1:200 risk of miscarriage. ${ }^{1}$ If there is no spinal fluid leak, serum $\alpha$-fetoprotein will be negative and neural defects will not be detected until much later in the pregnancy or after birth. At that point parents are faced with 3 options: voluntary abortion, postnatal closure, or, more recently, corrective in utero surgery, which is considered experimental. Three sites in the United States perform this procedure. ${ }^{3}$ 


\section{Conclusion}

Current methods of identifying neural tube defects are limited to second-trimester evaluations and may involve risk to the fetus. With the advent of ultrafast T2-weighted magnetic resonance imaging, visualization of spinal cord anomalies are possible earlier; however, the indication for this procedure is sonographic visualization of abnormal bony structures, which are typically not identified on 2-dimensional ultrasound during the first trimester. Literature reviews demonstrate that, to date, meningomyeloceles have not been identified on ultrasound within the first trimester. MedLine searches reveal that the most common time for identification of neural tube deficits is during the second and third trimester; the earliest defect was identified at 22 weeks gestational age. Even in the third trimester it is uncertain whether these lesions are meningomyeloceles or sacrococcygeal teratomas. 3-dimensional ultrasound allows for increased resolution of fetal surface anatomy, resulting in improved differentiation between fetal structures and early identification of developmental pathology.
The ability of 3-dimensional ultrasound to identify MMC at 9 weeks' gestation has the potential to bring about a revision in the guidelines for congenital defect screening. For some, early detection offers a simpler option to terminate a pregnancy whereas, for others, early detection offers hope for early intervention and a potentially improved longterm prognosis.

\section{References}

1. ACOG Committee on Practice Bulletins. ACOG practice bulletin. Clinical management guidelines for obstetrician-gynecologists. Number 44, July 2003 (Replaces Committee Opinion Number 252, March 2001). Obstet Gynecol 2003;102:203-13.

2. Jobe AH. Fetal surgery for myelomeningocele. N Engl J Med 2002;347:230-1.

3. Fichter MA, Dornseifer U, Henke J, et al. Fetal spina bifida repair: current trends and prospects of intrauterine neurosurgery. Fetal Diagn Ther 2008;23: 271-86.

4. Shaer CM, Chescheir N, Schulkin J. Myelomeningocele: a review of the epidemiology. Genetics, risk factors for conception, prenatal diagnosis, and prognosis for affected individuals. Obstet Gynecol Surv 2007;62:471-9. 